\title{
Variation on growth and yield traits among selected Phaseolus vulgaris landraces in KwaZulu-Natal, South Africa
}

\author{
VALENCIA VUYISILE NDLANGAMANDLA, NONTUTHUKO ROSEMARY NTULI ${ }^{\boldsymbol{\nu}}$ \\ Department of Botany, Faculty of Science and Agriculture, University of Zululand. Empangeni, Private Bag X1001, KwaDlangezwa 3886, South Africa. \\ Tel.: +27(0) 35902 6105. `email: NtuliR@unizulu.ac.za.
}

Manuscript received: 4 April 2019. Revision accepted: 14 May 2019

\begin{abstract}
Ndlangamandla VV, Ntuli NR. 2019. Variation on growth and yield traits among selected Phaseolus vulgaris landraces in KwaZulu-Natal, South Africa. Biodiversitas 20: 1597-1605. Phaseolus vulgaris L. (common bean) of American origin is grown worldwide for edible leaves, immature pods and dry seeds. This is the first comprehensive study conducted on variation among $P$. vulgaris landraces in South Africa. This study aimed to characterize variability in morpho-agronomic traits of $P$. vulgaris landraces. Twenty landraces were planted in a randomized complete block design with three replications. Variations in germination percentage as well as in stem, leaf, pod and seed traits were determined. Significant variations were recorded in all vegetative and reproductive traits except germination percentage and seed thickness. Vegetative traits correlated positively with each other, whereas reproductive traits correlated positively with both traits. Positive association of almost all traits with first and second components in a principal component analysis and biplot indicated them as potential discriminatory traits for landraces. The biplot and dendrogram associated landraces mainly according to their seed colour as well as growth and yield traits. This study revealed the potential vegetative and reproductive traits that can be used to select vigorously growing and high-yielding P. vulgaris landraces for future large-scale farming and breeding in South Africa. These traits could potentially result in desired plants with big stems, many branches, and numerous and broad leaves with high chlorophyll content, which will yield many, long and wide pods as well as many and heavy seeds. The taller landraces $(D$ $50 M 50 \mathrm{LB}-\mathrm{Cl}, \mathrm{D}-50 \mathrm{P} 50 \mathrm{C}-\mathrm{Cl}, \mathrm{E}-100 \mathrm{YG}-\mathrm{Cl}, \mathrm{E}-25 \mathrm{YG}-\mathrm{Cu}, \mathrm{E}-50 \mathrm{M} 50 \mathrm{LB}-\mathrm{Cu}, \mathrm{E}-50 \mathrm{~B} 50 \mathrm{C}-\mathrm{Cl}$ and $\mathrm{E}-50 \mathrm{DB} 50 \mathrm{C}-\mathrm{Cl}$ ) from KwaZulu-Natal show good potential for future large-scale farming and breeding for vigorous growth as well as high pod and seed yield.
\end{abstract}

Keywords: Durban, Eshowe, Phaseolus vulgaris landraces, reproduction traits, vegetative traits

\section{INTRODUCTION}

Phaseolus vulgaris L. is an important food legume in the Fabaceae family (Romero-Arenas et al. 2013). It is commonly known as the common bean, string bean, field bean, French bean and kidney bean (Musango et al. 2016). It provides a cheap source of protein to people in developing countries. $P$. vulgaris is the most nutritional crop because of its high protein content and plentiful amounts of fiber, provides vital nutrients and complex carbohydrates (Hegay et al. 2013). Landraces are variety of plants domesticated from the wild through natural and artificial selection (Abdollahi et al. 2016). Landraces helps farmers or agricultural programmes to adapt to new challenges such as climate change (Stoilova et al. 2013).

The germination of $P$. vulgaris is both hypogeal and epigeal (Musango et al. 2016). P. vulgaris is an annual herbaceous plant, climber or erect and some landraces show bushy growth types. The plant height of common bean landraces ranges from 19.5-123.4 cm during vegetative growth. Plants with the climbing growth habit grow taller and produce numerous branches (Stoilova et al. 2005). The bushy bean varieties are characterized by shorter plants when compared to the climbing varieties (Checa et al. 2006).
The leaf colour varies from dark to light green and the shape of the middle leaflet ranges from rhomboid to oval (Hornakova et al. 2003). The flower colour differs from white, cream, scarlet, red, yellow, white pink, purple to light purple (Romero-Arenas et al. 2013 ; Musango et al. 2016 ). The pods are either slender, curved or straight, longer and narrow. Curved pods are generally shorter in size (about $6 \mathrm{~cm}$ long) and straight pods are longer (about $13 \mathrm{~cm}$ ). The pod colour varies from black, purple, green to red. The most common seed colours of the $P$. vulgaris are white, red, yellow, brown, black, purple and grey. The seed shape varies from round, oval, elliptical, rhomboid, kidneyshaped to cylindrical (Romero-Arenas et al. 2013). Number of pods and seeds per plant are important determinants of yield in P. vulgaris (Stoilova et al. 2013).

Phaselous vulgaris crop is broadly grown from seeds saved from the previous harvest in South Africa including the KwaZulu-Natal province. Few studies have documented diversity of morpho-agronomic traits among $P$. vulgaris landraces elsewhere, but no literature has recorded such variation in South Africa. Thus this study focused on the variability in growth and yield traits among $P$. vulgaris landraces, which will form a baseline for future large-scale farming and breeding of these locally-adapted landraces. 


\section{MATERIALS AND METHOD}

\section{Seed sourcing and experimental design}

Seeds of $P$. vulgaris landraces were collected from Durban (Umbumbulu: 29.9846 ${ }^{\circ} \mathrm{S}, 30.7041^{\circ} \mathrm{E}$ ) and Eshowe (Khangelani: $29.0106^{\circ} \mathrm{S}, 31.2211^{\circ} \mathrm{E}$ ), in southern and northern KwaZulu-Natal, South Africa, respectively. Table 1 describes the 20 landraces used in this study, whose names are coined from the: area of collection percentage of seed coat colour - seed shape. The study was conducted at the University of Zululand, KwaDlangezwa campus, Orchard Unit farm $\left(28.8524^{\circ} \mathrm{S}, 31.8491^{\circ} \mathrm{E}\right)$. Representatives of $20 \mathrm{P}$. vulgaris landraces were planted in a randomised complete block design with three replications. Experimental plots were one meter long and $40 \mathrm{~cm}$ apart. Each plot had four rows $60 \mathrm{~cm}$ long, with inter-plant spacing of $10 \mathrm{~cm}$ and inter-row spacing of 20 $\mathrm{cm}$.

\section{Data collection}

Data on qualitative and quantitative characteristics were collected from five randomly selected plants in the inner two rows in order to eliminate border effects (Musango et al. 2016). The germination percentage was recorded at 14 days of planting. Other vegetative traits were measured at 21 days after planting (prior to flowering). However, the number of branches was counted at harvest, in order to minimize interference with flowering period. Qualitative traits recorded included growth habit; colour of stems, leaves, leaf veins, flowers and pods; as well as pod shape.

Table 1. Common bean landraces, source of origin and qualitative seed traits of Phaseolus vulgaris

\begin{tabular}{|c|c|c|c|}
\hline Landraces & $\begin{array}{c}\text { Area of } \\
\text { collection }\end{array}$ & $\begin{array}{c}\text { Seed coat colour } \\
\text { percentage }\end{array}$ & Shape \\
\hline D-50M50LB-Cl & Durban & $50 \% \mathrm{M}-50 \% \mathrm{LB}$ & $\mathrm{Cl}$ \\
\hline$D-50 P 50 C-C l$ & Durban & $50 \% \mathrm{P}-50 \% \mathrm{C}$ & $\mathrm{Cl}$ \\
\hline D-90M1OLLB-Cl & Durban & $90 \% \mathrm{M}-10 \%$ LLB & $\mathrm{Cl}$ \\
\hline D-90MIOSLB-Cl & Durban & $90 \% \mathrm{M}-10 \%$ SLB & $\mathrm{Cl}$ \\
\hline$D-90 C 10 L R-C l$ & Durban & $90 \% \mathrm{C}-10 \% \mathrm{LR}$ & $\mathrm{Cl}$ \\
\hline$D-100 Y G-C l$ & Durban & $100 \% \mathrm{YG}$ & $\mathrm{Cl}$ \\
\hline$D-90 B 10 C-C l$ & Durban & $90 \% \mathrm{~B}-10 \% \mathrm{C}$ & $\mathrm{Cl}$ \\
\hline$D-90 P 10 L B-K$ & Durban & $90 \% \mathrm{P}-10 \% \mathrm{LB}$ & $\mathrm{K}$ \\
\hline$D-50 L R 50 C-K$ & Durban & $50 \%$ LR-50\%C & K \\
\hline $\mathrm{E}-100 Y \mathrm{Y}-\mathrm{Cl}$ & Eshowe & $100 \% \mathrm{YG}$ & $\mathrm{Cl}$ \\
\hline$E-50 Y G-C l$ & Eshowe & $50 \% \mathrm{YG}$ & $\mathrm{Cl}$ \\
\hline$E-25 Y G-C u$ & Eshowe & $25 \% \mathrm{YG}$ & $\mathrm{Cu}$ \\
\hline E-90DP10C-Cu & Eshowe & $90 \% \mathrm{DP}-10 \% \mathrm{C}$ & $\mathrm{Cu}$ \\
\hline E-50P50C-Cu & Eshowe & $50 \% \mathrm{P}-50 \% \mathrm{C}$ & $\mathrm{Cu}$ \\
\hline E-50LP50C-Cu & Eshowe & $50 \%$ LP-50\% C & $\mathrm{Cu}$ \\
\hline$E-50 L P 50 C-K$ & Eshowe & $50 \%$ LP-50\% C & $\mathrm{K}$ \\
\hline E-50M50LB-Cu & Eshowe & $50 \% \mathrm{M}-50 \% \mathrm{LB}$ & $\mathrm{Cu}$ \\
\hline$E-50 B 50 C-C l$ & Eshowe & $50 \% \mathrm{~B}-50 \% \mathrm{C}$ & $\mathrm{Cl}$ \\
\hline$E-100 B k-C l$ & Eshowe & $100 \% \mathrm{Bk}$ & $\mathrm{Cl}$ \\
\hline E-50DB50C-Cl & Eshowe & $50 \% \mathrm{DB}-50 \% \mathrm{C}$ & $\mathrm{Cl}$ \\
\hline
\end{tabular}

Note: Landraces' names are currently unique to authors and are coined from: area of collection - percentage of seed coat colour(s) - seed shape. Seed colour: B. Brown, Bk. Black, C. Cream, DB. Dark brown, DP. Dark purple, LB. Light brown, LLB. Light brown (large), LG. Light green, LP. Light purple; M. Maroon, P. Purple, LR. Light red, RP. Reddish purple, YG. Yellowish green. Shape: Cl. Cylindrical, Cu. Cuboidal, K. Kidney.
Plant height $(\mathrm{mm})$ was measured from the scar of cotyledonous leaves to shoot apex using a ruler. Stem girth $(\mathrm{mm})$ was measured between the scar of the cotyledonuous leaves and the first set of true leaves, using Vernier callipers (Nassar et al. 2010). Number of leaves per plant was determined by direct counting. The chlorophyll content $\left(\mathrm{mg} \mathrm{cm}{ }^{-2}\right.$ ) was measured on the second fully-opened trifoliate leaf from the apex using CCM-200 plus chlorophyll meter. In each leaf lobe two readings were taken to calculate an average value for each plant (Richardson et al. 2002; Pereyra et al. 2014). The leaf area (length x width) $\left(\mathrm{mm}^{2}\right)$ was measured using a ruler.

The number of days to first flower and pod as well as $50 \%$ flower and pod formation were recorded (Bagheri et al. 2017). The number of pods per plant was counted directly. Pod length $(\mathrm{mm})$ and width $(\mathrm{mm})$ were each measured with Vernier callipers. The number of seeds per plant was determined by direct counting. Seed length $(\mathrm{mm})$, width $(\mathrm{mm})$ and thickness $(\mathrm{mm})$ were each measured using Vernier callipers. Total and 100-seed mass (g) was weighed using a Mettler PC 2000 weighing scale.

\section{Statistical analysis}

Data were analysed using GenStat $15^{\text {th }}$ edition for quantitative characteristics. The means of the different traits were compared using Tukey's 95\% confidence intervals test $(P<0.05)$. Variability of quantitative traits between and within landraces was evaluated by calculating the coefficient of variation. Correlation, principal component analysis, biplots and agglomerative hierarchical clustering (dendrogram) among traits were determined using XLSTAT.

\section{RESULTS AND DISCUSSION}

\section{Characteristics of the landraces}

Phaseolus vulgaris landraces showed high variability $(P<0.001)$ for most of the quantitative traits based on the analysis of variance (Tables 2 and 3). However, the CV values were relatively small for most of the measured traits. In general, the highest levels of variation were found for total seed mass $(\mathrm{CV}=52.2 \%)$ and seed thickness $(\mathrm{CV}=$ $51.3 \%$ ), whereas the lowest were in days to first flower formation $(\mathrm{CV}=3.9)$ and seed length $(\mathrm{CV}=5.9 \%)$.

The highest (87.0\%) and the lowest (33.3\%) germination percentages were recorded for landraces $E$ $50 Y G-C l$ and $\mathrm{E}-100 \mathrm{YG}-\mathrm{Cl}$, respectively (Table 2). The majority $(60 \%)$ of landraces had a bushy growth habit whereas the minority $(40 \%)$ had a climbing growth form. Landrace $E-25 Y G-C u$ had the tallest stems $(151.9 \mathrm{~mm})$ and $E-50 L P 50 C-K$ had the shortest stems $(77.7 \mathrm{~mm})$. Landraces E-50M5OLB-Cu and E-50DB5OC-Cl had the thickest stems $(5.2 \mathrm{~mm})$ whereas $\mathrm{D}-90 \mathrm{~B} 10 \mathrm{C}-\mathrm{Cl}$ had the thinnest stems $(3.4 \mathrm{~mm}$ ). Plants of $E-50 M 50 L B-C u$ produced numerous branches (2.7) whereas $\mathrm{D}-90 \mathrm{~B} 10 \mathrm{C}-\mathrm{Cl}, \mathrm{E}-50 \mathrm{P} 50 \mathrm{C}-\mathrm{Cu}$ and $E$-50LP50C-K produced fewer branches (1.7). The stems were green and hairy for all landraces whereas those of $E$ 25YG-Cu, E-50LP5OC-K and E-50M5OLB-Cu of Eshowe origin, and $\mathrm{D}-100 \mathrm{YG}-\mathrm{Cl}$ of Durban origin were maroon. 
Most landraces had green and hairy leaves, but $E$ $25 Y G-C u$ and E-50M50LB-Cu from Eshowe had dark green leaves (Table 2). Leaf veins were silver-green for almost all landraces except $E-50 L P 50 C$ - $K$ from Eshowe, which had maroon veins. Landraces $E-50 Y G-C l$ and $E$ 50M50LB-Cu produced numerous leaves (16.8) whereas $D$ $100 \mathrm{YG}-\mathrm{Cl}$ produced fewer leaves (8.5). Landrace $E$ $100 Y G-C l$ had the broadest leaves $\left(12753 \mathrm{~mm}^{2}\right)$ and $D$ $90 \mathrm{~B} 10 \mathrm{C}-\mathrm{Cl}$ had the narrowest leaves $\left(2364 \mathrm{~mm}^{2}\right)$. The highest leaf chlorophyll content $\left(28.5 \mathrm{mg} \mathrm{cm} \mathrm{cm}^{-2}\right)$ was recorded in $E-50 M 50 L B-C u$ and the lowest $\left(7.5 \mathrm{mg} \mathrm{cm}^{-2}\right)$ in $\mathrm{D}-90 \mathrm{ClOLR}-\mathrm{Cl}$.

Flower colour varied from a white, cream, $100 \%$ purple, $50 \%$ purple to $25 \%$ purple among the landraces. The earliest first flower (30 days after planting (DAP) and pod (37 DAP) formation was recorded in $E-100 B k-C l$ whereas $D$-50LR50C-K recorded the most delayed first flower (37 DAP) and pod (46 DAP) formation (Table 3). Landraces $\mathrm{D}-50 \mathrm{M} 50 \mathrm{LB}-\mathrm{Cl}$ and $\mathrm{E}-100 \mathrm{Bk}-\mathrm{Cl}$ were the earliest (32 DAP) to $50 \%$ flowering; $D-50 M 50 L B-C l, D$ $50 P 50 C-C l$ and $D-90 M 10 L L B-C l$ were the earliest to form $50 \%$ pods (42 DAP), whereas $D-50 L R 50 C$ - $K$ was the latest (40 and 49 DAP, respectively) in both cases.

Pod colour varied from green with purple strips, yellowish-green with pink strips, maroon to purple. Pod shape also varied, from straight, semi-curled to curled. Landrace $E-25 Y G-C u$ had numerous (13 pods per plant) pods whereas $D-50 L R 50 C$ - $K$ had the fewest (4 pods per plant). Landrace $\mathrm{E}-100 \mathrm{YG}-\mathrm{Cl}$ had the longest $(121 \mathrm{~mm})$ pods but $E$-50LP50C-Cu had the shortest $(83 \mathrm{~mm})$. Landrace $E-50 M 50 L B-C u$ had the widest $(11.6 \mathrm{~mm})$ pods whereas $E$-50LP50C-Cu had the narrowest $(9.5 \mathrm{~mm})$.

Landrace $E-25 Y G-C u$ had numerous seeds ( 55 seeds per plant) whereas $D-50 L R 50 C$ - $K$ had fewer seeds (18 seeds per plant) (Table 3). Plants of E-50M50LB-Cu had the biggest value for the total seed mass $(16.4 \mathrm{~g})$ whereas $D$ $90 M 10 L L B-C l$ had the lowest value $(8.0 \mathrm{~g})$. Landrace $D$ 50M50LB-Cl had the highest 100 -seed mass (47.4 g) whereas $E-90 D P 10 C-C u$ had the least $(15.0 \mathrm{~g})$. Plants of $D-50 M 50 L B-C l$ had the longest $(15.2 \mathrm{~mm})$ and the widest (7.9 mm) seeds whereas $\mathrm{E}-100 \mathrm{Bk}-\mathrm{Cl}$ had the shortest $(7.5$ $\mathrm{mm})$ and the narrowest $(5.2 \mathrm{~mm})$ seeds (Table 3$)$. Landrace $E-50 M 50 L B-C u$ produced the thickest $(6.8 \mathrm{~mm})$ seeds whereas $D$-90M10SLB-Cl and D-90C10LR-Cl produced the thinnest $(4.4 \mathrm{~mm})$.

Landraces from the same origin with different seed coat colour, intensity and shape differed in almost all measured traits (Tables 1; 2 and 3). In landraces from Eshowe area, $E$-50M5OLB-Cu either alone or in combination with others had higher growth and yield attributes compared with other landraces. Significant differences were also recorded among landraces from Durban, where in most cases $D$ $50 M 50 \mathrm{LB}-\mathrm{Cl}$ and/or $\mathrm{D}-50 \mathrm{P} 50 \mathrm{C}-\mathrm{Cl}$ outperformed other landraces.

Table 2. Variation in vegetative traits among Phaseolus vulgaris landraces

\begin{tabular}{|c|c|c|c|c|c|c|c|c|c|c|c|}
\hline Landrace & GP & GF & PH & SG & SC & NB & NL & LA & $\mathrm{CC}$ & $\mathbf{L C}$ & $\mathbf{L V}$ \\
\hline$D-50 M 50 L B-C l$ & $53.7^{\mathrm{a}}$ & $\mathrm{Bu}$ & $123.9^{\mathrm{abc}}$ & $4.2^{\mathrm{b}-\mathrm{e}}$ & $\mathrm{Gr}$ & $2.2^{\mathrm{ab}}$ & $10.8^{\mathrm{de}}$ & $4815^{\mathrm{bcd}}$ & $11.2^{\mathrm{fgh}}$ & $\mathrm{Gr}$ & $\mathrm{SGr}$ \\
\hline$D-50 P 50 C-C l$ & $65.7^{\mathrm{a}}$ & $\mathrm{Cb}$ & $117.1^{\mathrm{a}-\mathrm{d}}$ & $4.3^{\mathrm{b}-\mathrm{e}}$ & $\mathrm{Gr}$ & $2.3^{\mathrm{ab}}$ & $13.5^{\mathrm{a}-\mathrm{d}}$ & $6321^{\mathrm{b}}$ & $16.6^{\mathrm{b}-\mathrm{f}}$ & $\mathrm{Gr}$ & SGr \\
\hline$D-90 M 10 L L B-C l$ & $57.7^{\mathrm{a}}$ & $\mathrm{Bu}$ & $84.5^{\mathrm{cd}}$ & $4.2^{\text {cde }}$ & $\mathrm{Gr}$ & $1.7^{\mathrm{b}}$ & $10.6^{\mathrm{de}}$ & $5057^{\mathrm{bc}}$ & $9.6^{\mathrm{gh}}$ & $\mathrm{Gr}$ & SGr \\
\hline$D-90 M 10 S L B-C l$ & $58.1^{\mathrm{a}}$ & $\mathrm{Bu}$ & $87.9^{\mathrm{cd}}$ & $3.7^{\mathrm{ef}}$ & $\mathrm{Gr}$ & $2.1^{\mathrm{ab}}$ & $12.2^{\mathrm{b}-\mathrm{e}}$ & $4873^{\mathrm{bcd}}$ & $11.8^{\mathrm{fgh}}$ & $\mathrm{Gr}$ & SGr \\
\hline$D-90 C 10 L R-C l$ & $59.9^{\mathrm{a}}$ & $\mathrm{Cb}$ & $92.7^{\mathrm{cd}}$ & $4.1^{\mathrm{c}-\mathrm{f}}$ & $\mathrm{Gr}$ & $1.8^{\mathrm{b}}$ & $14.3^{\mathrm{a}-\mathrm{d}}$ & $5671^{\mathrm{bc}}$ & $7.5^{\mathrm{h}}$ & $\mathrm{Gr}$ & SGr \\
\hline$D-100 Y G-C l$ & $80.0^{\mathrm{a}}$ & $\mathrm{Bu}$ & $87.3^{\mathrm{cd}}$ & $4.1^{\mathrm{def}}$ & $\mathrm{Gr}$ & $2.3^{\mathrm{ab}}$ & $8.5^{\mathrm{e}}$ & $4397^{\mathrm{bcd}}$ & $13.6^{\mathrm{d}-\mathrm{g}}$ & $\mathrm{Gr}$ & SGr \\
\hline$D-90 B 10 C-C l$ & $71.3^{\mathrm{a}}$ & $\mathrm{Bu}$ & $98.9^{\mathrm{bcd}}$ & $3.4^{\mathrm{f}}$ & $\mathrm{Gr}$ & $1.9^{\mathrm{ab}}$ & $11.2^{\text {de }}$ & $2364^{\mathrm{d}}$ & $9.0^{\text {gh }}$ & $\mathrm{Gr}$ & SGr \\
\hline$D-90 P 10 L B-K$ & $72.3^{\mathrm{a}}$ & $\mathrm{Bu}$ & $93.8^{\text {bcd }}$ & $3.7^{\mathrm{ef}}$ & $\mathrm{Gr}$ & $1.87^{\mathrm{ab}}$ & $11.9^{\text {cde }}$ & $4283^{\mathrm{bcd}}$ & $11.5^{\mathrm{fgh}}$ & $\mathrm{Gr}$ & SGr \\
\hline$D-50 L R 50 C-K$ & $71.3^{\mathrm{a}}$ & $\mathrm{Bu}$ & $103.7^{\text {bcd }}$ & $3.7^{\mathrm{ef}}$ & $\mathrm{Gr}$ & $1.9^{\mathrm{ab}}$ & $11.0^{\mathrm{de}}$ & $3443^{\text {cd }}$ & $12.8^{\mathrm{e}-\mathrm{h}}$ & $\mathrm{Gr}$ & SGr \\
\hline$E-100 Y G-C l$ & $33.3^{\mathrm{a}}$ & $\mathrm{Cb}$ & $137.0^{\mathrm{ab}}$ & $4.8^{\mathrm{abc}}$ & $\mathrm{Gr}$ & $2.3^{\mathrm{ab}}$ & $16.2^{\mathrm{ab}}$ & $12753^{a}$ & $19.9^{\mathrm{bc}}$ & $\mathrm{Gr}$ & SGr \\
\hline $\mathrm{E}-50 Y \mathrm{Y}-\mathrm{Cl}$ & $87.0^{\mathrm{a}}$ & $\mathrm{Bu}$ & $122.4^{\mathrm{abc}}$ & $4.7^{\mathrm{a}-\mathrm{d}}$ & $\mathrm{Gr}$ & $2.3^{\mathrm{ab}}$ & $16.8^{\mathrm{a}}$ & $12526^{a}$ & $21.3^{\mathrm{b}}$ & $\mathrm{Gr}$ & SGr \\
\hline$E-25 Y G-C u$ & $78.7^{\mathrm{a}}$ & $\mathrm{Cb}$ & $151.9^{\mathrm{a}}$ & $5.0^{\mathrm{ab}}$ & M & $2.5^{\mathrm{ab}}$ & $16.1^{\mathrm{abc}}$ & $10991^{\mathrm{a}}$ & $18.0^{\mathrm{b}-\mathrm{e}}$ & DGr & SGr \\
\hline$E-90 D P 10 C-C u$ & $68.0^{\mathrm{a}}$ & $\mathrm{Cb}$ & $91.5^{\mathrm{cd}}$ & $3.7^{\mathrm{ef}}$ & $\mathrm{M}$ & $2.1^{\mathrm{ab}}$ & $14.5^{\mathrm{a}-\mathrm{d}}$ & $4590^{\mathrm{bcd}}$ & $9.8^{\text {gh }}$ & $\mathrm{Gr}$ & SGr \\
\hline E-50P50C-Cu & $81.0^{\mathrm{a}}$ & $\mathrm{Bu}$ & $113.1^{\mathrm{a}-\mathrm{d}}$ & $4.0^{\text {def }}$ & $\mathrm{Gr}$ & $1.7^{\mathrm{b}}$ & $11.3^{\mathrm{de}}$ & $4407^{\mathrm{bcd}}$ & $9.0^{\mathrm{gh}}$ & $\mathrm{Gr}$ & SGr \\
\hline$E-50 L P 50 C-C u$ & $76.7^{\mathrm{a}}$ & $\mathrm{Cb}$ & $104.3^{\mathrm{bcd}}$ & $3.7^{\mathrm{ef}}$ & $\mathrm{Gr}$ & $2.0^{\mathrm{ab}}$ & $12.1^{\mathrm{b}-\mathrm{e}}$ & $3170^{\text {cd }}$ & $12.9^{e-h}$ & $\mathrm{Gr}$ & SGr \\
\hline$E-50 L P 50 C-K$ & $44.0^{\mathrm{a}}$ & $\mathrm{Cb}$ & $77.7^{\mathrm{d}}$ & $4.3^{\mathrm{b}-\mathrm{e}}$ & $\mathrm{M}$ & $1.7^{\mathrm{b}}$ & $12.2^{\mathrm{b}-\mathrm{e}}$ & $4419^{\mathrm{bcd}}$ & $11.6^{\mathrm{fgh}}$ & $\mathrm{Gr}$ & $\mathrm{M}$ \\
\hline E-50M5OLB-Cu & $65.7^{\mathrm{a}}$ & $\mathrm{Cb}$ & $148.8^{\mathrm{a}}$ & $5.2^{\mathrm{a}}$ & $\mathrm{M}$ & $2.7^{\mathrm{a}}$ & $16.8^{\mathrm{a}}$ & $11715^{\mathrm{a}}$ & $28.5^{\mathrm{a}}$ & DGr & SGr \\
\hline $\mathrm{E}-50 \mathrm{~B} 50 \mathrm{C}-\mathrm{Cl}$ & $73.7^{\mathrm{a}}$ & $\mathrm{Bu}$ & $124.7^{\mathrm{abc}}$ & $4.7^{\mathrm{a}-\mathrm{d}}$ & $\mathrm{Gr}$ & $2.3^{\mathrm{ab}}$ & $13.9^{\mathrm{a}-\mathrm{d}}$ & $12294^{\mathrm{a}}$ & $19.6^{\mathrm{bcd}}$ & $\mathrm{Gr}$ & SGr \\
\hline$E-100 B k-C l$ & $46.7^{\mathrm{a}}$ & $\mathrm{Bu}$ & $98.8^{\mathrm{bcd}}$ & $3.7^{\mathrm{ef}}$ & $\mathrm{Gr}$ & $2.2^{\mathrm{ab}}$ & $14.4^{\mathrm{a}-\mathrm{d}}$ & $3674^{\text {cd }}$ & $14.1^{\mathrm{c}-\mathrm{g}}$ & $\mathrm{Gr}$ & SGr \\
\hline$E-50 D B 50 C-C l$ & $72.3^{\mathrm{a}}$ & $\mathrm{Bu}$ & $125.3^{\mathrm{abc}}$ & $5.2^{\mathrm{a}}$ & $\mathrm{Gr}$ & $2.5^{\mathrm{ab}}$ & $13.8^{\mathrm{a}-\mathrm{d}}$ & $11675^{\mathrm{a}}$ & $20.8^{b}$ & $\mathrm{Gr}$ & $\mathrm{SGr}$ \\
\hline Mean & 66.2 & & 109.3 & 4.2 & & 2.1 & 13.1 & 6672 & 14.5 & & \\
\hline P-value & 0.1 & & $<.001$ & $<.001$ & & $<.001$ & $<.001$ & $<.001$ & $<.001$ & & \\
\hline $\mathrm{CV} \%$ & 26.9 & & 30.6 & 13.8 & & 32.4 & 24.6 & 30.2 & 32.0 & & \\
\hline
\end{tabular}

Landrace are explained in Table 1. Traits: GP. Germination percentage (\%), GF. Growth form, PH. Plant height (mm), SG. Stem girth $(\mathrm{mm}), \mathrm{NB}$. Number of branches, NL. Number of leaves, CC. chlorophyll content $\left(\mathrm{mg} \mathrm{cm}^{-2}\right)$, LA. Leaf area ( $\left.\mathrm{mm}^{2}\right)$, SC. Stem colour, LC. Leaf colour, LV. Leaf veins. Growth form (GF): Bu. Bushy, Cb. Climbing. Stem colour (SC): Gr. Green, M. Maroon. Leaf colour (LC): DGr. Dark green, Gr .green. Leaf veins (LV): M. Maroon, SGr. Sliver green. Means followed by different letter(s) within a column differ significantly $(\mathrm{P}<0.05)$. 
Table 3. Variation in reproductive traits among Phaseolus vulgaris landraces

\begin{tabular}{|c|c|c|c|c|c|c|c|c|c|c|c|c|c|c|c|c|}
\hline ace & DFF & $\mathbf{5 0 \%} \mathbf{F}$ & FC & DPF & $\mathbf{5 0 \%} \mathrm{P}$ & PC & PS & $\mathbf{P}$ & PL & PW & NS & TSM & HSM & SL & SW & ST \\
\hline$O L B-C l$ & $1.0^{\mathrm{b}}$ & $32.0^{\mathrm{b}}$ & W & $39.0^{\mathrm{ab}}$ & $42.0^{\mathrm{ab}}$ & GPS & $\mathrm{S}$ & b-g & $11.3^{\mathrm{a}-\mathrm{e}}$ & $.5^{\mathrm{a}-\mathrm{e}}$ & $32.8^{\mathrm{a}-\mathrm{e}}$ & $12.7^{\mathrm{abc}}$ & $47.4^{\mathrm{a}}$ & $5.2^{\mathrm{a}}$ & $7.9^{\mathrm{a}}$ & $5.8^{\mathrm{a}}$ \\
\hline$D-50 P 50 C-C l$ & $31.3^{\mathrm{b}}$ & $34.7^{\mathrm{ab}}$ & $25 \% \mathrm{P}$ & $39.7^{\mathrm{ab}}$ & $42.0^{\mathrm{ab}}$ & GPS & $\mathrm{S}$ & $10.4^{\mathrm{a}-\mathrm{e}}$ & $113.5^{\mathrm{a}-\mathrm{d}}$ & $11.2^{\mathrm{ab}}$ & $42.1^{\mathrm{a}-\mathrm{d}}$ & $13.0^{\mathrm{abc}}$ & $35.5^{\mathrm{e}-\mathrm{h}}$ & $12.2^{\mathrm{d}-\mathrm{g}}$ & $6.7^{\mathrm{c}}$ & $5.4^{\mathrm{a}}$ \\
\hline$D-90 M 10 L L B-C l$ & $31.0^{\mathrm{b}}$ & $32.7^{\mathrm{b}}$ & $\mathrm{C}$ & $39.0^{\mathrm{ab}}$ & $42.0^{\mathrm{ab}}$ & YGpS & $\mathrm{S}$ & $5.8^{\mathrm{fg}}$ & $99.5^{\mathrm{c}-\mathrm{f}}$ & $10.5^{\mathrm{a}-\mathrm{e}}$ & $19.8^{\mathrm{de}}$ & $8.0^{\mathrm{cd}}$ & $41.1^{\mathrm{bcd}}$ & $13.6^{\mathrm{bc}}$ & $6.7^{\mathrm{cd}}$ & $5.0^{\mathrm{a}}$ \\
\hline$D-90 M 10 S L B-C l$ & $31.0^{\mathrm{b}}$ & $33.7^{\mathrm{ab}}$ & $\mathrm{C}$ & $41.0^{\mathrm{ab}}$ & $43.3^{\mathrm{ab}}$ & GPS & $\mathrm{S}$ & $7.9^{\mathrm{c}-\mathrm{g}}$ & $97.9^{\text {efg }}$ & $10.3^{\mathrm{b}-\mathrm{e}}$ & $33.1^{\mathrm{a}-\mathrm{e}}$ & $10.0^{\mathrm{a}-\mathrm{d}}$ & $30.8^{\text {hi }}$ & $11.1^{\mathrm{g}}$ & $5.7^{\mathrm{ef}}$ & $4.4^{\mathrm{a}}$ \\
\hline$D-90 C 10 L R-C l$ & $32.3^{b}$ & $33.6^{\mathrm{ab}}$ & $\mathrm{C}$ & $41.3^{\mathrm{ab}}$ & $44.7^{\mathrm{ab}}$ & YGpS & $S$ & $7.5^{\text {efg }}$ & $116.4^{\mathrm{ab}}$ & $10.3^{\mathrm{b}-\mathrm{e}}$ & $37.0^{\mathrm{a}-\mathrm{e}}$ & $11.5^{\mathrm{abc}}$ & $36.2^{\mathrm{d}-\mathrm{g}}$ & $12.1^{\mathrm{efg}}$ & $5.8^{\text {def }}$ & $4.4^{\mathrm{a}}$ \\
\hline$D-1$ & $31.0^{\mathrm{b}}$ & $35.0^{\mathrm{ab}}$ & $\mathrm{C}$ & $.0^{\mathrm{ab}}$ & $43.3^{\mathrm{ab}}$ & GPS & SC & $7.5^{\mathrm{d}-\mathrm{g}}$ & $5.7^{\mathrm{fg}}$ & & & $9.2^{\mathrm{a}-\mathrm{d}}$ & $41.7^{\mathrm{bc}}$ & $12.0^{\mathrm{efg}}$ & $7.1^{\mathrm{abc}}$ & $6.1^{\mathrm{a}}$ \\
\hline$D-C$ & 3 & $35.3^{\mathrm{ab}}$ & $\mathrm{C}$ & & $44.7^{\mathrm{ab}}$ & GPS & SC & & efg & & & $8.6^{\mathrm{bcd}}$ & $34.2^{\mathrm{e}-\mathrm{h}}$ & $12.9^{\text {cde }}$ & $6.6^{\text {cde }}$ & $5.5^{\mathrm{a}}$ \\
\hline$B-K$ & $32.7^{b}$ & $34.7^{\mathrm{ab}}$ & $\mathrm{P}$ & $.0^{\mathrm{ab}}$ & $43.3^{\mathrm{ab}}$ & GPS & $\mathrm{S}$ & & $98.2^{\mathrm{d}-\mathrm{g}}$ & $9.7^{\mathrm{de}}$ & $32.6^{\mathrm{a}-\mathrm{e}}$ & $10.3^{\mathrm{a}-\mathrm{d}}$ & $42.8^{\mathrm{ab}}$ & $14.3^{\mathrm{ab}}$ & $7.6^{\mathrm{ab}}$ & $6^{\mathrm{a}}$ \\
\hline & & 40. & $\mathrm{C}$ & & 49 & GPS & $\mathrm{S}$ & & & $11.2^{\mathrm{ab}}$ & $18.1^{\mathrm{e}}$ & $4.0^{\mathrm{d}}$ & $31.0^{\text {ghi }}$ & $12.7^{\mathrm{c}-\mathrm{f}}$ & $6.8^{\mathrm{bc}}$ & $8^{\mathrm{a}}$ \\
\hline & & 34.0 & $\mathrm{C}$ & & 39. & GPS & $\mathrm{S}$ & & & & & $12.5^{\mathrm{abc}}$ & $31.4^{\mathrm{f}-\mathrm{i}}$ & $12.1^{\mathrm{efg}}$ & $6.7^{\mathrm{bc}}$ & $4^{\mathrm{a}}$ \\
\hline & & $35.0^{\mathrm{ab}}$ & $\mathrm{C}$ & & $43.3^{\mathrm{ab}}$ & GPS & S & $2^{\mathrm{abc}}$ & $4^{\mathrm{ab}}$ & $\mathrm{abc}$ & $\mathrm{ab}$ & $14.8^{\mathrm{abc}}$ & $33.9^{\mathrm{e}-\mathrm{i}}$ & $13.5^{\mathrm{bc}}$ & $7.4^{\mathrm{abc}}$ & $5.3^{\mathrm{a}}$ \\
\hline & & $35.0^{\mathrm{ab}}$ & $\mathrm{P}$ & & $43.3^{\mathrm{ab}}$ & GPS & S & & $.2^{\mathrm{a}}$ & 11 & & $15.6^{\mathrm{ab}}$ & $28.7^{\mathrm{i}}$ & $11.6^{\mathrm{fg}}$ & $7.1^{\mathrm{abc}}$ & $5.1^{\mathrm{a}}$ \\
\hline$C u$ & $3^{\mathrm{b}}$ & $35.0^{\mathrm{ab}}$ & $\mathrm{P}$ & ab & $43.7^{\mathrm{ab}}$ & $\mathrm{P}$ & $\mathrm{S}$ & & $\mathrm{fg}$ & & $32.3^{\mathrm{b}-\mathrm{e}}$ & $9.1^{\mathrm{a}-\mathrm{d}}$ & $15.0^{\mathrm{k}}$ & $1^{g}$ & $6.7^{\mathrm{cd}}$ & $5.3^{\mathrm{a}}$ \\
\hline & $0^{\mathrm{b}}$ & $35.0^{\mathrm{ab}}$ & $\mathrm{P}$ & $3^{\mathrm{ab}}$ & $44.0^{\mathrm{ab}}$ & GPS & S & 7. & $103.7^{\mathrm{b}-\mathrm{f}}$ & $11.0^{\mathrm{abc}}$ & $25.4^{\text {cde }}$ & $8.9^{\text {bcd }}$ & $37.4^{\text {cde }}$ & $13.4^{\mathrm{bcd}}$ & $7.2^{\mathrm{abc}}$ & $6.1^{\mathrm{a}}$ \\
\hline$C u$ & $7^{\mathrm{b}}$ & $35.0^{\mathrm{ab}}$ & $\mathrm{P}$ & $0^{\mathrm{ab}}$ & $45.3^{\mathrm{ab}}$ & M & $\mathrm{C}$ & $12.5^{\mathrm{ab}}$ & $2^{g}$ & $9.5^{\mathrm{e}}$ & $40.5^{\mathrm{a}-\mathrm{e}}$ & $8.8^{\mathrm{bcd}}$ & $19.7^{\mathrm{jk}}$ & $9.6^{\mathrm{h}}$ & $5.8^{\text {def }}$ & $4.9^{\mathrm{a}}$ \\
\hline$C-K$ & $3^{\mathrm{b}}$ & $34.3^{\mathrm{ab}}$ & $\mathrm{P}$ & $0^{\mathrm{ab}}$ & $42.7^{\mathrm{ab}}$ & GDPS & $\mathrm{S}$ & $9.3^{\mathrm{a}-\mathrm{f}}$ & $89.7^{\text {fg }}$ & $10.4^{\mathrm{b}-\mathrm{e}}$ & $33.8^{\mathrm{a}-\mathrm{e}}$ & $9.1^{\mathrm{a}-\mathrm{d}}$ & $31.3^{\mathrm{f}-\mathrm{i}}$ & $11.5^{\mathrm{fg}}$ & $7.0^{\mathrm{abc}}$ & $5.8^{\mathrm{a}}$ \\
\hline$O L B-C u$ & $32.7^{\mathrm{b}}$ & $35.0^{\mathrm{ab}}$ & $50^{\circ}$ & $1.0^{\mathrm{ab}}$ & $43.3^{\mathrm{ab}}$ & GPS & S & $12.0^{\mathrm{a}-\mathrm{d}}$ & $115.9^{\mathrm{ab}}$ & $11.6^{\mathrm{a}}$ & $52.0^{\mathrm{ab}}$ & $16.4^{\mathrm{a}}$ & $36.6^{\mathrm{c}-\mathrm{f}}$ & $13.1^{\text {cde }}$ & $6.9^{\mathrm{bc}}$ & $6.8^{\mathrm{a}}$ \\
\hline $\mathrm{OC}-\mathrm{Cl}$ & $32.0^{\mathrm{b}}$ & $33.7^{\mathrm{ab}}$ & $\mathrm{W}$ & $40.3^{\mathrm{ab}}$ & $43.3^{\mathrm{ab}}$ & GPS & S & $9.9^{a-f}$ & $113.7^{\mathrm{abc}}$ & $10.7^{\mathrm{a}-\mathrm{d}}$ & $39.5^{\mathrm{a}-\mathrm{e}}$ & $11.1^{\mathrm{a}-\mathrm{d}}$ & $30.5^{\mathrm{hi}}$ & $12.9^{\text {cde }}$ & $7.0^{\mathrm{abc}}$ & $5.6^{\mathrm{a}}$ \\
\hline $\mathrm{E}-100 \mathrm{Bk}-\mathrm{Cl}$ & $30.0^{\mathrm{b}}$ & $32.0^{\mathrm{b}}$ & $50 \% \mathrm{P}$ & $37.0^{\mathrm{b}}$ & $42.0^{\mathrm{ab}}$ & GDPS & $\mathrm{SC}$ & $9.7^{\mathrm{a}-\mathrm{f}}$ & $92.0^{\mathrm{fg}}$ & $9.9^{\text {cde }}$ & $32.6^{\mathrm{a}-\mathrm{e}}$ & $11.9^{\mathrm{abc}}$ & $21.3^{\mathrm{j}}$ & $7.5^{\mathrm{i}}$ & $5.2^{\mathrm{f}}$ & $4.5^{\mathrm{a}}$ \\
\hline $\mathrm{E}-50 \mathrm{DB} 50 \mathrm{C}-\mathrm{Cl}$ & $30.3^{b}$ & $35.0^{\mathrm{ab}}$ & $\mathrm{C}$ & $40.0^{\mathrm{ab}}$ & $42.7^{\mathrm{ab}}$ & GPS & $S$ & $10.8^{\mathrm{a}-\mathrm{e}}$ & $117.2^{\mathrm{ab}}$ & $10.8^{\mathrm{a}-\mathrm{d}}$ & $42.9^{\mathrm{abc}}$ & $11.9^{\mathrm{abc}}$ & $32.4^{\mathrm{e}-\mathrm{i}}$ & $13.1^{\text {cde }}$ & $7.1^{\mathrm{abc}}$ & $5.5^{\mathrm{a}}$ \\
\hline $\mathrm{Me}$ & 31.8 & 34.6 & & 40.6 & 43.4 & & & 9.2 & 104.4 & 10. & 36.0 & 10.9 & 32.9 & 12.3 & 6.8 & 8.5 \\
\hline & $<.001$ & 0.068 & & 0.110 & 0.217 & & & $<$. & $<.001$ & $<.001$ & $<.001$ & $<.001$ & $<.001$ & $<.001$ & $<.001$ & 0.442 \\
\hline $\mathrm{CV} \%$ & 3.9 & 6.4 & & 6.3 & 6.3 & & & 37.0 & 11.3 & 8.5 & 47.7 & 52.2 & 10.1 & 5.9 & 8.4 & 51.3 \\
\hline
\end{tabular}

Note: Landrace are explained in Table 1. Traits: DFF. Days to first flowering, 50\% F. Days to 50\% flowering, FC. Flower colour, DPF. Days to pod formation, 50\% P. Days to 50\% pod formation, PC. Pod colour, PS. Pod shape, PL. Pod length (mm), PW. Pod width (mm), NP. Number of pods per plant, NS. Number of seeds per plant, TSM. Total seed mass (g), HSM. Hundred seed mass (g), SL. Seed length (mm), SW. Seed width (mm), ST. Seed thickness (mm). Flower colour (FC): C. Cream, 25; 50 and 100\%P - 25; 50 and 100\% purple, W. White. Pod colour (PC): GDPS. Green with dark purple strips, GPS. Green with purple strips, M. Maroon, P. Purple, YGpS. Yellowish green with pink strips. Pod shape (PS): C. Curled, S. Straight, SC. Semi-curled. Means followed by different letter(s) within a column differ significantly $(\mathrm{P}<0.05)$

Furthermore, landraces with the same seed coat colour but from different origins differed in some traits. Plants sown from $100 \%$ yellowish-green seeds originating from the Eshowe area $(E-100 Y G-C l)$ were taller; with thicker stems; had numerous and broader leaves with higher chlorophyll content; as well as longer pods than plants from the Durban $(D-100 Y G-C l)$. A landrace with $50 \%$ maroon, 50\% light brown seeds originating from Eshowe area $(E-50 M 50 L B-C u)$ had thicker stems; numerous and broader leaves with higher chlorophyll content than a similar landrace from the Durban area $(D-50 M 50 L B-C l)$. However, $\mathrm{D}-50 \mathrm{M} 5 \mathrm{LB}-\mathrm{Cl}$ of Durban origin had longer seeds and heavier 100-seeds than E-50M50LB-Cu of Eshowe origin. The same trend was recorded in 100-seed weight between $\mathrm{D}-100 \mathrm{YG}-\mathrm{Cl}$ and $\mathrm{E}-100 \mathrm{YG}-\mathrm{Cl}$ of Durban and Eshowe origins, respectively. Leaves of $D-50 P 50 C-C l$ with $50 \%$ purple, $50 \%$ cream seeds originating from Durban had higher chlorophyll content than a similar landrace from Eshowe $(E-50 P 50 C-C u)$.

\section{Correlation among morpho-agronomic traits}

Only positive correlations were significant among the measured morpho-agronomic traits (Table 4). Plant height correlated positively with stem girth, number of branches, number of leaves, leaf area, chlorophyll content, number of pods, pod length, pod width, number of seeds per plant and total seed mass. Leaf area and chlorophyll content correlated positively with each other and also correlated with stem girth, number of branches and number leaves. Stem girth correlated positively with number of branches, pod length, pod width, number of seeds per plant and total seed mass. Chlorophyll content and number of pods correlated positively with each other and they both correlated positively with number of branches, number of leaves and leaf area.

A positive correlation was recorded between the days to first pod formation and 50\% pod formation and both further correlated positively with days to first flower formation and $50 \%$ flowering. There was also a positive correlation between days to first flower formation and days to $50 \%$ flowering. Pod length and width correlated positively with each other. The number of seeds per plant correlated positively with the total seed mass and they both positively correlated with number of branches, number of leaves, leaf area, chlorophyll content, number of pods and pod length. Seed length correlated positively with seed width. Seed width and seed thickness also correlated positively with each other. 
Table 4. Correlation of morpho-agronomic traits among Phaseolus vulgaris landraces

\begin{tabular}{|c|c|c|c|c|c|c|c|c|c|c|c|c|c|c|c|c|c|c|}
\hline Traits & GP & PH & SG & NB & NL & LA & $\mathrm{CC}$ & DFF & $\mathbf{5 0 \%} \mathbf{F}$ & DFP & $\mathbf{5 0 \%} \mathrm{P}$ & NP & PL & PW & SL & SW & ST & NS \\
\hline$\overline{\mathrm{PH}}$ & 0.141 & & & & & & & & & & & & & & & & & \\
\hline SG & 0.015 & 0.748 & & & & & & & & & & & & & & & & \\
\hline NB & 0.129 & 0.752 & 0.684 & & & & & & & & & & & & & & & \\
\hline NL & -0.101 & 0.642 & 0.598 & 0.565 & & & & & & & & & & & & & & \\
\hline LA & 0.056 & 0.771 & 0.908 & 0.708 & 0.744 & & & & & & & & & & & & & \\
\hline $\mathrm{CC}$ & 0.087 & 0.766 & 0.812 & 0.854 & 0.664 & 0.842 & & & & & & & & & & & & \\
\hline DFF & 0.186 & 0.006 & -0.166 & -0.261 & -0.121 & -0.156 & -0.056 & & & & & & & & & & & \\
\hline $50 \% \mathrm{~F}$ & 0.447 & 0.057 & -0.092 & -0.041 & -0.090 & -0.062 & 0.086 & 0.815 & & & & & & & & & & \\
\hline DFP & 0.289 & -0.119 & -0.341 & -0.314 & -0.289 & -0.286 & -0.191 & 0.848 & 0.833 & & & & & & & & & \\
\hline $50 \% \mathrm{P}$ & 0.529 & -0.225 & -0.418 & -0.310 & -0.300 & -0.406 & -0.272 & 0.761 & 0.756 & 0.819 & & & & & & & & \\
\hline NP & 0.084 & 0.611 & 0.574 & 0.679 & 0.751 & 0.623 & 0.662 & -0.390 & -0.187 & -0.430 & -0.378 & & & & & & & \\
\hline PL & 0.009 & 0.756 & 0.793 & 0.572 & 0.612 & 0.826 & 0.577 & -0.178 & -0.187 & -0.289 & -0.433 & 0.388 & & & & & & \\
\hline PW & 0.154 & 0.605 & 0.643 & 0.500 & 0.352 & 0.586 & 0.580 & 0.238 & 0.369 & 0.081 & -0.052 & 0.152 & 0.603 & & & & & \\
\hline SL & 0.241 & 0.225 & 0.277 & -0.036 & -0.171 & 0.228 & 0.063 & 0.230 & 0.112 & 0.197 & -0.009 & -0.296 & 0.436 & 0.353 & & & & \\
\hline SW & 0.314 & 0.327 & 0.381 & 0.144 & -0.104 & 0.296 & 0.202 & 0.159 & 0.164 & 0.008 & -0.101 & -0.019 & 0.347 & 0.415 & 0.829 & & & \\
\hline ST & 0.245 & 0.247 & 0.232 & 0.159 & -0.147 & 0.120 & 0.314 & 0.353 & 0.330 & 0.231 & 0.055 & -0.074 & 0.063 & 0.364 & 0.581 & 0.739 & & \\
\hline NS & 0.143 & 0.716 & 0.712 & 0.729 & 0.822 & 0.763 & 0.747 & -0.262 & -0.145 & -0.362 & -0.330 & 0.907 & 0.647 & 0.312 & -0.055 & 0.099 & -0.014 & \\
\hline TSM & -0.016 & 0.714 & 0.704 & 0.736 & 0.738 & 0.701 & 0.680 & -0.437 & -0.428 & -0.596 & -0.556 & 0.743 & 0.749 & 0.348 & 0.065 & 0.159 & 0.043 & 0.885 \\
\hline
\end{tabular}

Note: Traits are explained in Tables 2 and 3. Significant values $\geq 0.6$ are in bold.

Table 5. Principal component coefficients of traits for different Phaseolus vulgaris landraces

\begin{tabular}{|c|c|c|c|c|}
\hline Traits & PC1 & PC2 & PC3 & PC4 \\
\hline GP & 0.016 & 0.488 & -0.252 & 0.706 \\
\hline $\mathrm{PH}$ & 0.840 & 0.280 & -0.148 & -0.052 \\
\hline SG & 0.891 & 0.197 & 0.070 & -0.132 \\
\hline NB & 0.831 & 0.040 & -0.192 & 0.137 \\
\hline NL & 0.780 & -0.174 & -0.394 & -0.192 \\
\hline LA & 0.910 & 0.148 & -0.064 & -0.148 \\
\hline $\mathrm{CC}$ & 0.855 & 0.195 & -0.230 & -0.017 \\
\hline DFF & -0.336 & 0.725 & -0.399 & -0.289 \\
\hline $50 \% \mathrm{~F}$ & -0.232 & 0.726 & -0.583 & -0.046 \\
\hline DPF & -0.483 & 0.653 & -0.444 & -0.165 \\
\hline $50 \% \mathrm{P}$ & -0.540 & 0.506 & -0.564 & 0.123 \\
\hline NP & 0.776 & -0.296 & -0.355 & 0.302 \\
\hline PL & 0.825 & 0.188 & 0.211 & -0.254 \\
\hline PW & 0.551 & 0.556 & -0.059 & -0.308 \\
\hline SL & 0.137 & 0.713 & 0.589 & 0.008 \\
\hline SW & 0.275 & 0.678 & 0.494 & 0.255 \\
\hline ST & 0.135 & 0.712 & 0.266 & 0.221 \\
\hline NS & 0.886 & -0.098 & -0.257 & 0.184 \\
\hline TSM & 0.910 & -0.170 & 0.092 & 0.105 \\
\hline HSM & 0.030 & 0.465 & 0.788 & -0.049 \\
\hline Eigenvalue & 8.362 & 4.344 & 2.884 & 1.137 \\
\hline Variability (\%) & 41.810 & 21.722 & 14.422 & 5.685 \\
\hline Cumulative \%) & 41.810 & 63.532 & 77.954 & 83.640 \\
\hline
\end{tabular}

Note: Traits are explained in Tables 2 and 3. Significant values $\geq$ 0.6 are in bold.

\section{Principal component analysis}

The first four informative principal components explained $83.640 \%$ of the total variation (Table 5). The first component $(\mathrm{PC} 1)$ correlated positively with plant height, stem girth, number of branches, number of leaves, leaf area, chlorophyll content, number of pods, pod length, number of seeds and total seed mass that accounted for $41.810 \%$ of the total variation.

The second component (PC2) accounted for $21.722 \%$ of the total variation and was defined positively by days to first and 50\% flower formation; days to first pod formation; as well as seed length, width and thickness. Hundred-seed mass was positively correlated with the third component (PC3) that described $14.422 \%$ of the total variation. The fourth component (PC4) accounted for 5.685\% of the total variation and was positively associated with germination percentage.

\section{Cluster analysis}

In a biplot all traits correlated positively with either PC1, PC2 or both (Fig. 1). Biplot further clustered the landraces into six different groups. Group I was composed of E-100YG-Cl, E-5OYG-Cl, E-25YG-Cu and E-50M5OLB$\mathrm{Cu}$, all originating from the Eshowe area. Landraces with $100 \%$ yellowish-green $(E-100 Y G-C l), 50 \%$ yellowishgreen $(E-50 Y G-C l)$ and $25 \%$ yellowish-green $(E-25 Y G-C u)$ had similar seed coats, which differed only in colour intensity (Table 1). Group II contained D-50M50LB-Cl, D$50 P 50 C-C l, E-50 B 50 C-C l$ and $E-50 D B 50 C-C l$, where the first two landraces are of Durban origin and the last two are of Eshowe origin. Landrace $\mathrm{E}-50 \mathrm{~B} 5 \mathrm{OC}-\mathrm{Cl}$ with $50 \%$ brown, $50 \%$ cream and $\mathrm{E}-50 \mathrm{DB} 50 \mathrm{C}$ - $\mathrm{Cl}$ with $50 \%$ dark brown, $50 \%$ cream also shared similar seed coats but different colour intensity (Table 1). Group III was formed by $\mathrm{E}-100 \mathrm{Bk}-\mathrm{Cl}$. 


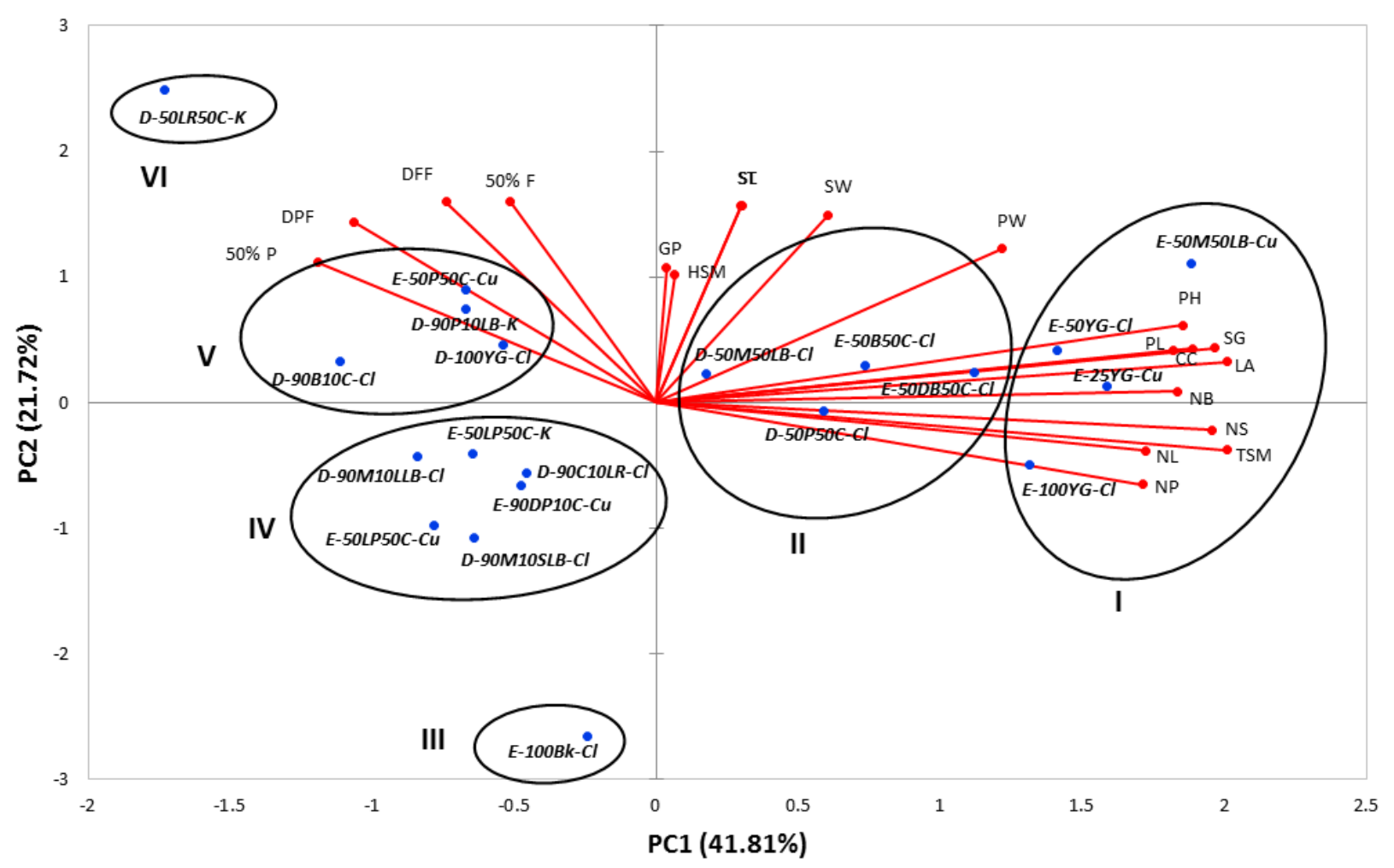

Figure 1. Biplot based on the first two principal component (PC) for morpho-agronomic traits and Phaseolus vulgaris landraces. Note: Description for landraces is in Table 1. Traits are explained in Tables 2 and 3

Group IV was composed of $D-90 M 10 L L B-C l, D$ 90M10SLB-Cl, D-90C10LR-Cl, E-90DP10C-Cu, E$50 L P 50 C-C u$ and $E-50 L P 50 C-K$, with the first and last three landraces originating from Durban and Eshowe, respectively. Landraces $D-90 M 10 L L B-C l$ and $D$ $90 \mathrm{M} 10 \mathrm{SLB}-\mathrm{Cl}$ with $90 \%$ maroon, $10 \%$ light brown were similar in seed coats however $D-90 M 10 L L B-C l$ had larger seeds than $D$-90M10SLB-Cl. Plants of landraces with $50 \%$ light purple, $50 \%$ cream seeds (E-50LP5OC-Cu and E$50 L P 50 C-K)$ also had similar seed coats but differed in seed shape (Table 1). Group V was composed of $D-100 Y G$ $C l, D-90 B 10 C-C l, D-90 P 10 L B-K$ from Durban and $E$ $50 P 50 C-C u$ from Eshowe. Group VI consisted of $D$ 50LR50C-K which separated on its own.

The relationship between the landraces illustrated by a dendrogram based on Euclidean distance grouped them into three main clusters (Fig. 2). Cluster I consisted of two sub-clusters. Sub-cluster I-A was composed of $E$ 90DP10C-Cu, E-50LP50C-Cu and E-100Bk-Cl, all from the Eshowe area. Sub-cluster I-B was further divided into two minor clusters. One of its minor clusters consisted of $D-90 M 10 L L B-C l, D-90 M 10 S L B-C l$ and D-90C10LR-Cl from Durban and $E-50 L P 50 C-K$ from Eshowe. Landraces $D-90 M 10 L L B-C l$ and D-90M1OSLB-Cl had the same $90 \%$ maroon, $10 \%$ light brown seed coats but D-90M10LLB-Cl had larger seeds than D-90M10SLB-Cl (Table 1). Another minor cluster comprised of D-50M50LB-Cl, D-100YG-Cl,
$D-90 B 10 C-C l$ and $D-90 P 10 L B-K$ from Durban and $E$ 50P50C-Cu from Eshowe.

Cluster II was composed of $E-100 Y G-C l, E-50 Y G-C l$, E-25YG-Cu, E-50M5OLB-Cu, E-50B50C-Cl and E$50 \mathrm{DB} 50 \mathrm{C}-\mathrm{Cl}$ from Eshowe and $\mathrm{D}-50 \mathrm{P} 50 \mathrm{C}-\mathrm{Cl}$ from Durban. Landrace $\mathrm{E}-100 Y G-C l, E-50 Y G-C l$ and $E-25 Y G-$ $\mathrm{Cu}$ had similar yellowish-green seed coats that differed in colour intensity, which was the similar case with $E$ $50 B 50 C-C l$ and $E-50 D B 50 C-C l$ with $50 \%$ brown, $50 \%$ cream seed coats (Table 1 ). Landrace $D-50 L R 50 C-K$ was on its own in Cluster III.

\section{Discussion}

Variation in morpho-agronomic traits

The same significant variations in plant height, days to first pod formation and pod length among $P$. vulgaris landraces in KwaZulu-Natal were found among corresponding landraces in Lages (Bertoldo et al. 2014), Turkey (Yeken et al. 2018) and Varanasi (Singh et al. 2017) for the same traits. This might indicate heterogeneity among these landraces, which is essential for breeding purposes. This heterogeneity is also true for the ranges in days to $50 \%$ flowering, number of seeds per plant and 100seed mass in these landraces, which are similar to those documented in Portugal and Bulgaria (Stoilova et al. 2005 and 2014). 


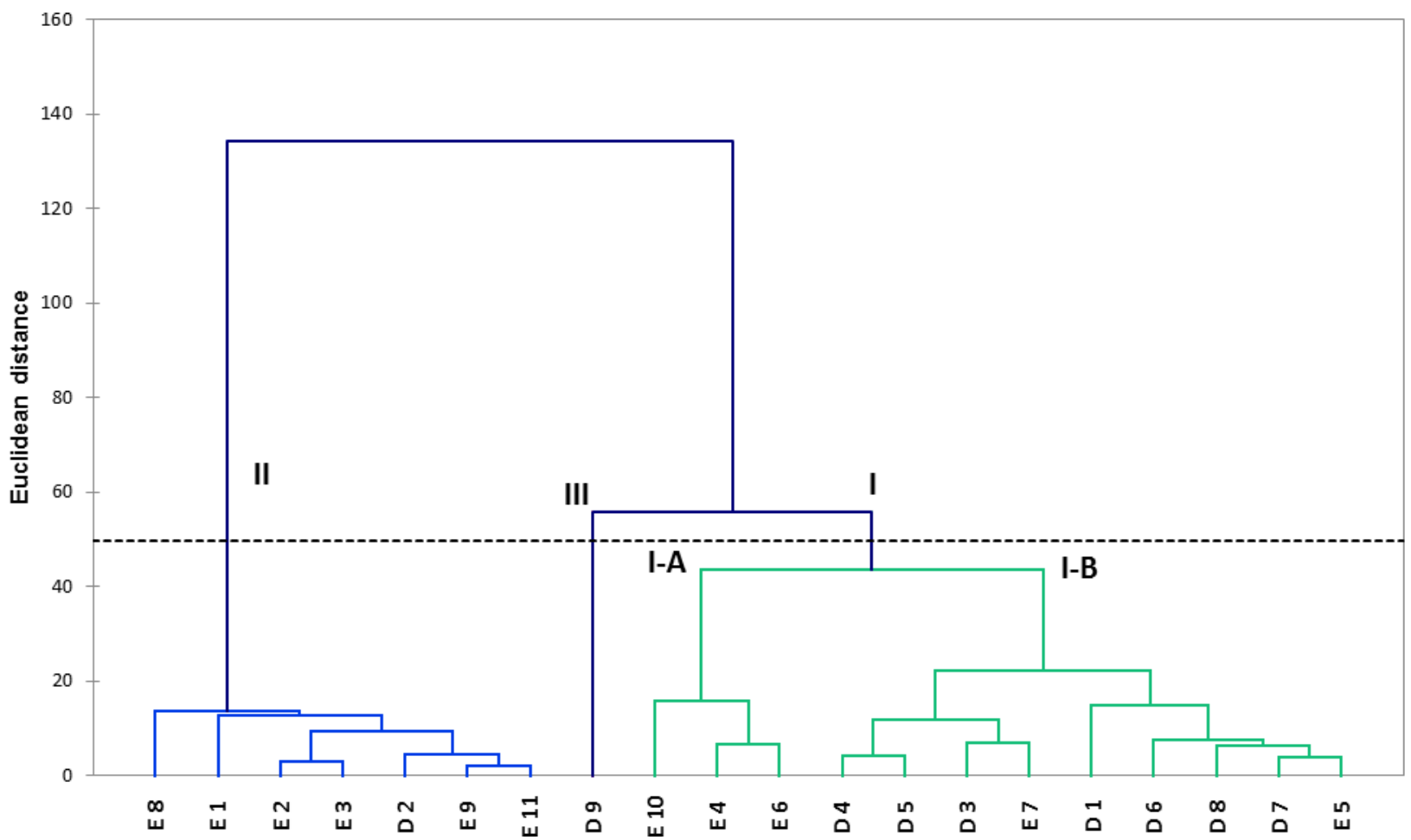

Figure 2. Dendrogram grouping of Phaseolus vulgaris landraces based on Euclidean distances. Note: Description for landraces is in Table 1. Landraces from Durban: D1, D-50M50LB-Cl; D2, D-50P50C-Cl; D3, D-90M10LLB-Cl; D4, D-90M10SLB-Cl; D5, D90C10LR-Cl; D6, D-100YG-Cl; D7, D-90B10C-Cl; D8, D-90P10LB-K; D9, D-50LR50C-K. Landraces from Eshowe: E1, E-100YG-Cl; E2, $E-50 Y G-C l ; \mathbf{E 3}, E-25 Y G-C u ; \mathbf{E 4}, E-90 D P 10 C-C u ; \mathbf{E 5}, E-50 P 50 C-C u ; \mathbf{E 6}, E-50 L P 50 C-C u ; \mathbf{E 7}, E-50 L P 50 C-K ; \mathbf{E 8}, E-50 M 50 L B-$ $\mathrm{Cu}$; E9, E-50B50C-Cl; E10, E-100Bk-Cl; E11, E-50DB50C-Cl

Smaller ranges in stem girth, number of leaves per plant and leaf area in the current study than those recorded among corresponding landraces in Egypt (Nassar et al. 2010) can be attributed to differences in types of landraces investigated as well as in the climatic conditions of these countries. This would be the same reason for the earlier formation of first flowers and $50 \%$ pods, and production of lighter total seeds per plant in landraces from KwaZuluNatal than those from Varanasi (Singh et al. 2017) and Zimbabwe (Musango et al. 2016), as well as Turkey (Yeken et al. 2018), respectively.

Differences among landraces with similar seed coat colour but different origins recorded in plant height $(D$ $100 Y G-C l$ and $E-100 Y G-C l$ ) and 100 -seed mass (D$50 M 50 L B-C l$ and $E-50 M 50 L B-C u ; D-100 Y G-C l$ and $E$ $100 Y G-C l$ ) were similar to those recorded among whiteseeded landraces from Yalova and Canakkale in Turkey (Yeken et al. 2018). Further, differences in 100-seed mass of landraces with the same origin, similar seed coat colour, but different sizes (D-90M10LLB-Cl and D-90M10SLB-Cl) and seed shape (E-50LP50C-Cu and E-50LP50C-K) were similarly recorded in white-seeded landraces $(\mathrm{CNK}-2$ and CNK-4) from Canakkale (Yeken et al. 2018).

Landraces E-25YG-Cu and E-50M5OLB-Cu represented the tallest, vigorously climbing plants with numerous branches, which were similar to the corresponding plants from Bulgaria and Portugal (Stoilova et al. 2005). Climbing bean varieties are characterized by tall stems, long internodes and climbing ability (Checa et al. 2006). Significant variation in stem girth of landraces with different seed coat colour (E-50LP50C-K and E-50DB50C$\mathrm{Cl}$ ) but from the same area was possibly caused by their genetic diversity, as reported by Chávez-Servia (2016).

Diversity in number of leaves on similar landraces with different origins (D-50M50LB-Cl and E-50M50LB-Cu; D$100 Y G-C l$ and $E-100 Y G-C l$ ) probably resulted from diverse environmental conditions of their locations. It is possible that landraces from the Durban area (D-50M50LB-Cl and $D-100 Y G-C l$ were previously growing under environmental stress, which caused leaf growth inhibition compared to their counterparts from the Eshowe area $(E$ $50 M 50 L B-C u$ and $E-100 Y G-C l)$, as was the case with landraces in Turkey (Kusvuran and Dasgan 2017). The chlorophyll content was higher in leaves with broader and dark green $(E-25 Y G-C u$ and $E-50 M 50 L B-C u)$ leaves than the landraces with narrower leaves. This reflects that chlorophyll content increases with leaf growth and development but decreases at the senescence stage (Pereyra et al. 2014) and under stress conditions such as drought (Kusvuran and Dasgan 2017).

The colours are responsible for the wide variation in $P$. vulgaris plants (Musango et al. 2016), where colours of 
shoots and petals are either controlled by one gene or closely linked genes (Okii et al. 2014). This would explain the production of $25 \%$ and $100 \%$ purple flowers from plants with maroon stems as well as cream, white and 25$100 \%$ purple flowers from plants with green stems. The purple flowers produced green pods with purple strips and also maroon pods. The cream flowers generated green pods with purple strips and also yellowish-green pods with pink strips. The white flowers produced only green pods with purple strips. This variation in flower and pod colours within landraces can be explained in terms of mutation and segregation (Musango et al. 2016).

Some landraces studied probably formed flowers and pods relatively early either because of genetic variation or as a result of stress. Plants escape environmental stress by channelling all their resources towards reproductive stages for fast maturity (Asfaw et al. 2013). Days to first flowering and length of flowering period vary depending on the landrace and the environmental conditions experienced (Peksen 2007). The production of numerous pods and seeds per plant by landrace $E-25 Y G-C u$ indicates a consistent and strong correlation between number of pods per plant and seed yield (Peksen 2007).

\section{Correlation in agronomic traits of $P$. vulgaris landraces}

A positive correlation of most vegetative (growth) traits among each other and also with yield traits, particularly number and size of pods as well as number and mass of seeds, concurs with the findings on landraces in Greece and Iran, where the number of pods per plant correlated positively with various traits (Akhshi et al. 2015; Bagheri et al. 2017). Correlation studies suggested that selection and/or breeding practices towards tall plants in South Africa will result in improved growth (stem girth, number of branches, number of leaves, leaf area and chlorophyll content) and yield (number length and width of pods as well as number and total mass of seeds) traits because of their positive correlation among each other.

A correlation between plant height and total seed mass was evident in the current study, where taller landraces had heavier seeds per plant $(>12 \mathrm{~g})$ whereas shorter ones had lighter seeds ( $<12 \mathrm{~g})$ (Tables 2 and 3$)$. The current findings were similar to those of $P$. vulgaris landraces in Nilgiris, where four dwarf plants had seed mass $<60.0 \mathrm{~g}$, whereas tall landraces had $>60.0 \mathrm{~g}$ (Jose et al. 2009). This suggests that the taller landraces $D-50 M 50 L B-C l, D-50 P 50 C-C l, E$ $100 Y G-C l, E-25 Y G-C u, E-50 M 50 L B-C u, E-50 B 50 C-C l$ and E-50DB50C-Cl from KwaZulu-Natal are potential candidates for future large-scale farming and breeding towards vigorous growth and high seed yield.

\section{Principal component and cluster analyses}

In the current study, PCA summarised the variation in morpho-agronomic traits into four principal components, where $\mathrm{PC} 1$ and $\mathrm{PC} 2$ contributed to high variability $(63.53 \%)$. Traits in these components are essential in differentiating landraces. PC1 incorporated almost all vegetative traits, including number of pods, pod length, number of seeds and total seed mass. Other essential traits in PC2 included days to flowering and first pod formation as well as seed size. These findings are similar to those of P. vulgaris landraces in Iran (Akhshi et al. 2015).

Association of landraces in a biplot and dendrogram was primarily based on their uniqueness in seed coat colour, similarity in agronomic traits and then on their origins. The isolate grouping of $D-50 L R 50 C-K$ in a biplot (Group VI) and dendrogram (Cluster III) could be that it delayed in flowering and pod formation, and also produced the fewest and the lightest seeds per plant (Fig. 1 and 2, Table 3). Landrace $E-100 B k-C l$, with black seeds, also formed a separate group in a biplot (Group III) but was associated with $\mathrm{E}-90 \mathrm{DP} 10 \mathrm{C}-\mathrm{Cu}$ and $\mathrm{E}-50 \mathrm{LP} 50 \mathrm{C}-\mathrm{Cu}$ in a dendrogram (Cluster I-A). Isolate clustering was possibly due to its earliest flowering and pod formation as well as its production of the shortest and narrowest seeds (Table 3), whereas its association with others was based on their common origin (Table 1). Apparently, landraces with black seeds form an operational taxonomic unit relatively distant from other landraces, probably due to their unique agromorphological characteristics (Jose et al. 2009).

The clustering of landraces with varying yellowishgreen seeds (E-100YG-Cl, E-50YG-Cl and E-25YG-Cu), and with $50 \%$ maroon, $50 \%$ light-brown seeds (E$50 M 50 L B-C u$ ) from the Eshowe area in Group I of a biplot and Cluster II of a dendrogram (including $\mathrm{D}-50 \mathrm{P} 50 \mathrm{C}-\mathrm{Cl}$, $E-50 B 50 C-C l$ and $E-50 D B 50 C-C l$ ) could have resulted from their similarity in seed coat colour and the common area of origin. Similar findings were recorded among $P$. vulgaris accessions in Zimbabwe (Musango et al. 2016), Nilgiris (Jose et al. 2009) and Turkey (Yeken et al. 2018). These landraces were probably also associated because they had the tallest plants with thicker stems, highest chlorophyll content, numerous branches, broader leaves, longer pods, numerous pods and seeds and also heavier seeds compared with other landraces (Table 2 and 3 ).

The close association of the remaining landraces in a biplot (Groups II, IV and V) and dendrogram (Cluster I-B) was based either on the seed coat colour, origin and/or similarities in growth and yield traits. In Zimbabwe, $P$. vulgaris landraces with different seed coats but from the same origin clustered together (Musango et al. 2016). Also, distinctiveness in traits of landraces causes them to cluster separately from each other (Balkaya and Ergün 2008), which might be the case with KwaZulu-Natal landraces.

Selection for cultivation of and breeding towards tall landraces in South Africa can result in plants with large stems, numerous branches, profuse and broad leaves with high chlorophyll content, which will yield numerous, long and wide pods with many and heavy seeds. These are the desired traits for human consumption of leaves as vegetables; pods as green beans; and seeds as cooked dry beans. Landraces $D-50 M 50 L B-C l, \quad D-50 P 50 C-C l, E-$ 10OYG-Cl, E-25YG-Cu, E-50M5OLB-Cu, E-50B50C-Cl and $E-50 D B 50 C-C l$, which were much taller, can be recommended for future breeding and large-scale farming.

In conclusion, effective improvement of landraces on growth and yield can be easily developed among $P$. vulgaris landraces from KwaZulu-Natal because vegetative and reproductive traits correlated positively with each other. Almost all studied growth and yield traits are 
appropriate for studying variability among landraces since they were positively associated with components of high variability (PC1 and PC2) in principal component analysis. Grouping of landraces in a biplot and dendrogram according to similarities in their seed coat colour as well as growth and yield traits enhances selection of vigorously growing and high yielding landraces for future large-scale farming and breeding. Therefore, breeding towards and large-scale farming of tall landraces in South Africa will result in plants with wide stems, many branches, numerous and broad leaves with high chlorophyll content, which will yield numerous, long and wide pods with many and heavy seeds. Thus, the selection of tall landraces $D-50 M 50 \mathrm{LB}-\mathrm{Cl}$, $D-50 P 50 C-C l, E-100 Y G-C l, E-25 Y G-C u, E-50 M 50 L B-C u$, $E-50 B 50 C-C l$ and $E-50 D B 50 C-C l$ from KwaZulu-Natal will possibly lead towards vigorously growing and high yielding accession.

\section{ACKNOWLEDGEMENTS}

This work was supported by the National Research Foundation (NRF) and the Botany department. The Department of Botany, University of Zululand, South Africa is thanked for providing land and other necessities for the experiments. Sincere gratitude is due to the University Research Office authorities, particularly Ms. Zinhle Ntuli, for granting time to prepare this manuscript.

\section{REFERENCES}

Abdollahi A, Tahmasebpour B, Dehghanian H. 2016. Factor analysis of phenological and morphological traits in common bean (Phaseolus vulgaris L.). Biol Forum 8 (1): 132-134.

Akhshi N, Firouzabadi FN, Cheghamirza K, Dorri HR. 2015. Coefficient analysis and association between morpho-agronomical characters in common bean (Phaseolus vulgaris L.). Cercetari Agronomice in Moldova 48 (4): 29-37.

Asfaw A, Almekinders CJM, Struik PC, Blair MW. 2013. Farmers' common bean variety and seed management in the face of drought and climate instability in southern Ethiopia. Academic J 8 (22): 1022 1037.

Bagheri M, Kahrizi D, Zebarjadi A. 2017. Study on genetic variation and morpho-phenologic traits in common bean (Phaselous vulgaris L.). Biharean Biol 11 (1): 43-47.

Balkaya A, Ergün A, 2008. Diversity and use of pinto bean (Phaseolus vulgaris) populations from Samsun, Turkey. New Zeal J Crop Hortic Sci 36 (3): 189-197.

Bertoldo JG, Coimbra JLM, Guidolin AF, Andrade LRBD, Nodari RO 2014. Agronomic potential of genebank landrace elite accessions for common bean genetic breeding. Sci Agr 71 (2): 120-125.
Chávez-Servia JL, Heredia-García E, Mayek-Pérez N, Aquino-Bolaños EN, Hernández-Delgado S, Carrillo-Rodríguez JC, Gill-Langarica HR, Vera-Guzmán AM. 2016. Diversity of common bean (Phaseolus vulgaris L.) landraces and the nutritional value of their grains. In Grain Legumes. InTech.

Checa O, Ceballos H, Blair MW. 2006. Generation means analysis of climbing ability in common bean (Phaseolus vulgaris L.). J Hered 97 (5): 456-65

Hegay S, Geleta M, Bryngelsson T, Asanaliev A, Garkava-Gustavsson L, Hovmalm HP, Ortiz R. 2013. Genetic diversity analysis in Phaseolus vulgaris L. using morphological traits. Genet Resour Crop Evol DOI 10.1007/s10722-013-0056-3.

Hornakova O, Zavodna M, Zakova M, Kraic J, Debre F. 2003. Diversity of common bean landraces collected in the western and eastern Carpatien. Czech J Genet Plant Breed 7 (3): 73-83.

Jose FC, Mohammed MS, Thomas G, Varghese G, Selvaraj N, Dorai M. 2009. Genetic diversity and conservation of common bean (Phaseolus vulgaris L., Fabaceae) landraces in Nilgiris. Curr Sci 97 (2): 227-235.

Kusvuran S, Dasgan HY. 2017. Effects of drought stress on physiological and biochemical changes in Phaseolus vulgaris L. Legume Res 40 (1): 55-62.

Musango R, Kudzai K, Mhungu S, Tibugar H. 2016. Phenotypic characterization of common bean (Phaseolus vulgaris L.) accessions conserved at the genetic resources and biotechnology institute. J Biodiv Environ Sci 8 (6): 26-36.

Nassar RMA, Ahmed YM, Boghdady MS. 2010. Botanical studies on Phaseolus vulgaris L. I-Morphology vegetative and reproductive growth. Int J Bot 6 (3): 323-333.

Okii D, Tukamuhabwa P, Kami J, Namayanja A, Paparu M, Ugen P, Gepts P. 2014. The genetic diversity and population structure of common bean (Phaseolus vulgaris L) germplasm in Uganda. Afr J Biotechnol 13 (29): 2935-2949.

Peksen E. 2007. Dynamics of flower appearance, flowering, pod and seed setting performance and their relations to seed yield in common bean (Phaseolus vulgaris L.). Pak J Bot 39 (2): 485-496.

Pereyra MS, Davidenco V, Núñez SB, Argüello JA. 2014. Chlorophyll content estimation in oregano leaves using a portable chlorophyll meter: relationship with mesophyll thickness and leaf age. Agron Ambiente 34 (1-2): 77-84.

Richardson AD, Duigan SP, Berlyn GP. 2002. An evaluation of noninvasive methods to estimate foliar chlorophyll content. New Phytol 153 (1): 185-194.

Romero-Arenas O, Damián Huato MA, Rivera Tapia JA, Báez Simón A, Huerta Lara M, Cabrera Huerta E. 2013. The nutritional value of Beans (Phaseolus vulgaris L.) and its importance for feeding of rural communities in Puebla-Mexico. Int Res J Biol Sci 2 (8): 59-65.

Singh V, Singh AK, Moharana DP, Singh B, Jaiswal DK, Singh DK. 2017. Assessment of quantitative differences among diverse genotypes of French bean (Phaseolus vulgaris L.) for yield and yield attributing traits. J Pharmacogn Phytochem 6 (4): 1557-1559.

Stoilova T, Pereira G, Tavares de Sousa MM, Carnide V. 2005. Diversity in common bean landraces (Phaseolus vulgaris L.) from Bulgaria and Portugal. J Cent Eur Agric 6 (4): 443-448.

Stoilova T, Pereira G, de Sousa M. 2013. Morphological characterization of a small common bean (Phaseolus vulgaris L.) collection under different environments. J Cent Eur Agric 14 (3): 854-864.

Stoilova T, Berova M, Kouzmova K, Stamatov S, 2014. Study on diversity of Phaseolus spp. landraces with reference to global climate change. Afr J Agr Res 9 (39): 2925-2935.

Yeken MZ, Kantar F, Çancı H, Özer G, Çiftçi V. 2018. Breeding of dry bean cultivars using Phaseolus vulgaris landraces in Turkey. Uluslararası Tarım ve Yaban Hayatı Bilimleri Dergisi 4 (1): 45-54. 